\title{
ESTIMATION OF CERTAIN COMPOUND AGAINST COTTON LEAFWORM, Spodoptera littoralis (BOISD.) ON SUGAR BEET PLANTS \\ Said, A. A. A. ${ }^{*}$; F. A. H. Shaheen ${ }^{*}$; E. A. H. Sherief ${ }^{* *}$ and H. A. M. Fouad ${ }^{\star *}$ \\ * Pesticides Dept., Fac. of Agric., Mansoura Univ, Egypt. \\ **Plant Protec. Res. Ins., A. R. C., Dokki-Giza, Egypt.
}

\begin{abstract}
A field study was conducted to compare the efficiency of the biocides, insecticides and insect growth regulators (IGR's) against cotton leafworm Spodoptera littoralis, whereas the results appeared that Virust was the most effective compound as it recording $63.64 \%$ reduction in the initial kill after five days achieved. Followed by Agerin and Brofect this gave 51.61, 46.38\% reduction. While, Protecto was the least efficient compound, as it gave $27.14 \%$ reduction only. but about insecticides as lannate and Cord in eleven days after treatment it have a high efficiency in reduction $(100 \%)$, while Flaxe was the lowest effective one which revealed $83.08 \%$ reduction. On the other hand IGR'S, Toporon and Runner were gave (88.87 and $88.44 \%$ reduction) and Demelin was the lowest effective (63.12\% reduction) after one day of treatment. As for the residual effect also Runner, Toporon, Virtue, keleron and Nomolt induced the best results, recording 100, 99.28, 99.08, 97.57 and 97.47 reduction percentages, respectively. Whereas, Demelin was the lowest effective one, which revealed $87.46 \%$ reduction during the period from $10^{\text {th }}$ to $15^{\text {th }}$ days after sprays application in the first season. While, in the second season Runner, Nomolt, Coragen and Virtue were the most effective insecticides they caused 85.73, 77.65, 77.17 and $73.01 \%$ reduction, after five days of spraying (initial kill), respectively. On the other hand, Demelin and Diberone induced a low residual effect, where it showed only $89.49 \%$ and $86.65 \%$ reduction after 15 days of treatment.
\end{abstract}

Key words: biocides, insecticides, IGR'S, Spodoptera littoralis and sugar beet.

\section{INTRODUCTION}

Sugar beet (Beta vulgaris L.) is one of the most important sugar crops in the world. It produces annually about $40 \%$ of sugar production all over the world. This crop is attacked with many insect pests throughout the growing season (Fouad et al. 2011). The Cotton leafworm larvae, Spodoptera littoralis (Boisd.) is considered as one of the important insect on sugar beet. It is active almost all the year round. It is a polyphagous insect having 112 host plants the larval stage feeding on many field and vegetable crop (Meisner and Nemny 1992, El-Maghraby et al. 1999, Khidr et al. 2003 and Sherief et al. 2009). Usually, this pest is controlled by using many conventional insecticides which often result many bad and undesirable side effects such as environmental pollution, resistance appearance in the target pests.

Radwan et al. (2004) used biocides for control S. littoralis; these materials may not cause any kind of pollution on the environment. Also, others reported from this investigation that the bioinsecticide (Xentari) demonstrated the least harmful effect on entomophagous insect population 
Said, A. A. A. et al.

which was significantly lower than those counted in control. The safety of bacterial bioinsecticides to insect predators was studied by Kares (1991) and to insect parasitoid population by Atwood et al. (1997).

The aim of this investigation is to evaluation the efficiency of certain chemical and bioinsecticides against $S$. littoralis on sugar beet plants in the field.

\section{MATERIALS AND METHODS}

This experiment was conducted at Kafr El-hamam village, Zagazig district, Sharkia Governorate, during 2009/2010 season. Experiment area $1680 \mathrm{~m}^{2}$. The sugar beet variety used was Baraca, sowed at 20 of September. This area divided into eight treatments. Each treatment has been divided into five replicates including control. The area of each replicate was $42 \mathrm{~m}^{2}\left(6 \times 7 \mathrm{~m}^{2}\right)$; so that the area of each treatment was $210 \mathrm{~m}^{2}\left(5 \times 42 \mathrm{~m}^{2}\right)$. Date of spray in 2 of November on sugar beet seedlings. Five sugar beet plants were chosen randomly from each replicate (25 plants per treatment) to estimate the number of $S$. littoralis larvae before spray and at the following dates 2, 5, 7, 9 and 11 days after spraying.

\section{The tested compounds:}

1-Biocides:

a. Agerin (6.5\% WP):

Common name: Bacillus thuringiensis Egypt. (B.t).

b. Protecto (9.4\% WP):

Common name: Bacillus thuringiensis kurstaki. (B.t).

c. Profect $(2+5 \%$ WP):

Common name: Spodoptera littoralis Nuclear Polyhydrosis Virus (NPV).

d. Virust:

+ Bacillus thuringiensis kurstaki (B.t).

Common name: Spodoptera littoralis Nuclear Polyhydrosis Virus (NPV).

\section{2-Insecticides:}

a. Lannate $(90 \% \mathrm{SP})$ :

Common name : methomyl.

Chemical name: S-methomyl $-N$-(methylcarbamoyloxy) thioacetimidate.

b. Cord (72\% EC):

Common name : profenofos.

Chemical name: O-(4-bromo-2-chlorophenyl) O-ethyl S-propyl phosphorothioate.

c. Radical (0.5\% EC):

Common name : emamectin benzoate.

Chemical name: (4"R)-5-O-demethyl-4"-deoxy-4"-(methyl amino) avermectin $\mathrm{A} 1 \mathrm{a}+(4 " R)$-5-O-demethyl-25-de (1-methylpropyl)-4"deoxy-4"-(methyl amino)-25-(1-methylethyl) avermectin

d. Flaxe (15\% SC): A1a $(9: 1)$.

Common name: indoxacarb. 
Chemical name: methyl (S)-7-chloro-2,5-dihydro-2-[[(methoxycarbonyl)[4 (trifluoromethoxy)phenyl]amino]carbonyl]indeno[1,e] $[1,3,4]$ oxadiazine-4a $(3 H)$-carboxylate.

\section{3-Insect growth regulator (IGR's):}

This experiment was conducted at Zagazig district, Sharkia Governorate, during the two successive seasons, 2009/2010 and 2010/2011. Experiment area $1680 \mathrm{~m}^{2}$. This area divided into eight treatments. Each treatment has been divided into five replicates including control. The area of each replicate was $42 \mathrm{~m}^{2}\left(6 \times 7 \mathrm{~m}^{2}\right)$; so that the total area of each treatment was $210 \mathrm{~m}^{2}(5 \mathrm{x}$ $\left.42 \mathrm{~m}^{2}\right)$. Each compound was used separately in a single treatment contain four replicates. Date of spray in 28 of October on sugar beet seedlings. Five sugar beet plants were chosen randomly from each replicate $(25$ plants per treatment) to estimate the number of $S$. littoralis larvae before spray and at the following dates 5,10 and 15 days after spraying.

Reduction percentage ( $\mathrm{R} \%$ ) was estimated according to the formula of Henderson and Tilton (1955) as follows:

$$
\% \mathrm{R}=1-\left(\frac{\text { Insect No in control before spray }}{\text { Insect No in control after spray }} X \frac{\text { Insect No in treatment after spray }}{\text { Insect No in treatment before spray }}\right) X 100
$$

\section{Statistical analysis}

Statistical analysis were carried out to determine the differences between treatments and days after spraying by using one way analysis of variance (ANOVA) (Costat, 1990) and Duncan's multiple range test (Duncan, 1955) was applied at $5 \%$ probability level.

\section{RESULTS AND DISCUSSION}

\section{Biocides and insecticides:}

Results are presented in Table (1) cleared that Virust was the most effective compound against the larvae. During the first five days after treatment $(63.64 \%$ reduction), followed by Agerin and Brofect that gave $51.61,46.38 \%$ reduction, while, Protecto was the least efficient compound, it gave $27.14 \%$ reduction only as initial kill after five days from treatment.

Residual effect of Protecto induced the best results, recording 77.05 of reduction percentages in $S$. littoralis, whereas, Brofect was the lowest effective one, which revealed $72.85 \%$ reduction during the period from $5^{\text {th }}$ to $11^{\text {th }}$ days after spray application.

All tested pesticides were significantly affected on population of the cotton leafworm $S$. littoralis at the indicated days after application Table (2). Data revealed that all the tested pesticides induced an obvious initial kill, where they exhibited from 38.69 to $81.04 \%$ reduction in population. Eleven days after treatment, Lannate and Cord gave a high efficiency in reduction the cotton leafworm population (100\%). While, Flaxe was the lowest effective one which revealed $83.08 \%$ reduction. 
Said, A. A. A. et al.

$1-2$ 
Concerning residual activity, the tested pesticides can be arranged in descending order as follows: Lannate, Cord, Radical and Flaxe recording 94.93, 92.39, 75.99 and $62.90 \%$ reduction, respectively.

Generally the second period during 2008/2009 season was not evaluated by $S$. littoralis because the population density was not reached to the level of economic threshold in the experimental area.

Another results near from data of Amal et al. (2003) reported that two biocides, Agerin and Ecotech at three concentrations were applied in the field on sugar beet plants to control Spodoptera littoralis. Where all treatments caused reduction in the numbers of $S$. littoralis larvae. Abo ElFtooh (2004) who mentioned that the pesticides Profenofos was more effective on $S$. littoralis than each of the isolated bacteria and the two bioinsecticides through the experiment period. Munir et al. (2005) found that Emamectin proved to be the best followed by Indoxacarb in their timeoriented mortality at three concentration levels tested. Abamectin proved to be the least effective to control this pest. Hassan et al. (2009) Found that efficacy of a new semi-synthetic avermectin derivative Methylamine avermectin (Radical $0.5 \%$ EC) was determined against larval instars of the Egyptian cotton leafworm, Spodoptera littoralis (Biosd.) in the laboratory, field and semi field experiments. $2^{\text {nd }}$ and $4^{\text {th }}$ instars larvae showed greatest susceptibility to the Radical in the laboratory experiment.

\section{Insect growth regulator (IGR's):}

Data in Table (3) cleared that Toporon and Runner was the most effective (88.87 and $88.44 \%$ reduction) Virtue, Diberone, Coragen and Keleron were moderately effective $(73.17,70.08,68.84$ and $68.12 \%$ reduction, respectively). While, Demelin was the lowest effective one and exhibited $63.12 \%$ reduction after one day of treatment.

Concerning the effect of the tested insecticides after 10 days of application, both Runner, Toporon and Virtue showed the highest effects (100, 98.56 and $98.16 \%$ reduction, respectively) followed by Keleron, Nomolt and Coragen (95.15, 94.95 and $93.95 \%$ reduction, respectively). However Demelin was the lowest active one and showed $85.24 \%$ reduction. Regarding to 15 days after application Runner, Nomolt, Toporon, Keleron and Virtue were not significantly different in their effect on reduction percentage of insect infestation (100\% for ever). While, Coragen, Diberone and Demelin gave $(95.01,90.99,89.68 \%$ reduction, respectively) during the first season. As for the residual effect also Runner, Toporon, Virtue, Keleron and Nomolt induced the best results, recording 100, 99.28, 99.08, 97.57 and 97.47 reduction percentages in $S$. littoralis. Whereas, Demelin was the lowest effective one, which revealed $87.46 \%$ reductions during the period from 10 th to 15 th days after spray treatment.

The results presented in the second season Table (4) Runner, Nomolt, Coragen and Virtue were the most effective insecticides they caused 85.73, $77.65,77.17$ and $73.01 \%$ reduction, after five days of spraying (initial kill), respectively. With the respect to residual effect, Runner was the highly effective recording $95.77 \%$ reduction in cotton leafworm population after 10 days of pesticides application. 
Said, A. A. A. et al.

3 
J. Plant Prot. and Path., Mansoura Univ., Vol. 3 (12), December, 2012

4 
On the other hand, Demelin and Diberone induced a low residual effect, where it showed only $89.49 \%$ and $86.65 \%$ reduction after 15 days of application. It could be concluded that, Runner, Toporon and Virtue had longest residual effect, recording 97.88, 95.53 and $95.33 \%$ reduction, respectively.

Our results agree with that obtained by Chandler et al. (1992) tested the toxic effects of three insect growth regulators (Di/lubenzuron, Te/lubenzuron, and Fenoxycarb) to 1- and 7-d-old larvae of corn earworm, Helicoverpa zea (Baddie), and fall armyworm, Spodoptera frugiperda (J. E. Smith). Fenoxycarb was more active against 1- and 7-d-old corn earworm larvae compared with $\mathrm{Di} /$ lubenzuron or Tef1ubenzuron; all compounds were equally active against 7-d-old fall armyworm larvae. Abd El-rahman et al. (2007) tested the direct and latent effects of the growth inhibitor Lefenuron and the combination of Lefenuron/Deltanet on the development of $S$. littoralis larvae. Both compounds proved to be toxic to the test insect larvae. Leufenuron proved to be more toxic than Leufenuron/Deltanet, $S$. littoralis larvae suffered from more mortality when they were fed for a longer period on the treated diet. Sammour et al. (2008) observed that the results also indicated mat Leufenuron was eliminated two times faster than Chlorfluazuron.

\section{REFERENCES}

Abd El-Rahman S.M.; E.M. Hegazy and A.E. Elwey (2007). Direct and latent effects of two chitin synthesis inhibitors to Spodoptera littoralis larvae (Boisd.). American-Eurasian J. Agric. \& Environ. Sci., (2007), 2(4): 457-464.

Abo El-Ftooh, A. A. (2004). Efficiency estimation of isolated bacteria Bacillus thuringiensis entomocidus against cotton leaf worm (Spodoptera littoralis Boisd) infesting sugar beet in comparison with two commercial bio-insecticide and insecticide Profenofos. Ann. of Agric. Sci, Moshtohor; 42(3): 1405-1413.

Amal A. El -Zoghbey; A. Atalla Fatma and A.H Mesbah (2003). Effect of two biocides in controlling Cassida vittata (Vill.) and Spodoptera littoralis (Boisd.) infesting sugar beet plants. Annals of Agric. Sci., Moshtohor, 41 (1): 339-346.

Atwood, D. W., S. Y. Young and T. J. Kring (1997). Impact of $B$. thuringiensis and thiodicarb alone and in combination on tobacco budworm, mortality and the cotton laefworm, Spodoptera littoralis (Boisd.) (Lepidoptera: Noctuidae). Egypt. J. Appl. Sci., 15 (12): 263-272.

Chandler, L. D.; S.D. Pair and J.R. Raulston (1992). Effects of selected insect growth regulators on longevity and mortality of Corn Earworm and Fall Armyworm (Lepidoptera: Noctuidae) Larvae. J. Econ. Entomology. 1992, 85(5): 1972-1978.

Costat Statistical Software (1990). Microcomputer program analysis version 4.20, Cohort Software, Berkeley, CA. 
Duncan, D.B. (1955). Multiple range and multiple F-tests. Biometrics.11:1-42.

El- Maghraby, H. M.; M. H .M. El- Kawalka; M.A. El-Bessomy and H. I. Omar (1999). Effect of three IGR's compound with chemical insecticides against cotton leafworm, Spodoptera littoralis (Boisd.) Infesting tomato plants. $2^{\text {nd }}$ intern at. Confer. Pest. Cont. Mansoura. Egypt. September, 29-34.

Fouad, H. A. M.; A. A. A. Said; F. A. H. Shaheen; E.A.H. Sherief (2011). Control some pests infesting sugar beet in sharkia governorate. M. Sc. Thesis, Fac. Agric. Mansoura Univ., 129 pp.

Hassan. F. Dahi; A. El-Sayed Yasser; M. El-Barkey Nehad; and F. Abd-El Aziz Mona (2009). Toxicological and biochemical studies of Methylamine Avermactin, a new type of bioinsecticide against the cotton leafworm, Spodoptera littoralis (Biosd). Egypt. Acad. J. biolog. Sci., 2 (1): 103- 116.

Henderson, C. F. and E.W. Tilton (1955). Test with acaricides against the brown wheat mite. J. Econ. Entomol. 84: 157-161.

Kares, E. A. (1991). Effect of mixtures of Bacillus thuringiensis (Berliner) and chemical insecticides against larvae of the pink bollworm, Pectinophora gossypiella (Saunders) (Lepidoptera: Gelechidae). Egypt. J. Biol. pest control, 1 (2): 15-23.

Khidr, A. A.; A. O. Abdeen; M. A. Eissa; A. M. H. Azab and A. I. Hassan (2003). Evaluation of some insecticides and their joint action against the cotton leafworm, Spodoptera littoralis (Boisd.) on lettuce vegetable plant, Lactuca sativa L. Ann. Agric. Sci. Moshtohor., 41 (3): 1323-1330.

Meisner, J. and N.E. Nemny (1992). The effect of margosano on the development of the Egyptian cotton leafworm, spodoptera littoralis (Boisd.) (Lepidoptera: Noctuidae). J. Appl. Entomol., 113 (4): 330-333.

Munir Ahmad, Mushtaq Ahmad Saleem and Mushtaq Ahmad (2005). Time oriented mortality in leafworm, Spodoptera litura (fab.) (Lepidoptera: noctuidae) by some new chemistry insecticides. Pak. Entomol., 27 (1), 67-70.

Radwan, S. M. E.; F. F. Shalaby; E. H. Abd El-Karim and E. S. Mansour (2004). The simultaneous effect of certain recommended insecticides and selective biocides on the changes in the population density of cotton leafworm and its related bio agents inhabiting cotton fields. Egypt. J. Agric. Res., 82 (3): 1139-1153.

Sammour E.A; M.A. Kandil and N.F. Abdel-Aziz (2008). The Reproductive Potential and Fate of Chlorfluazuron and Leufenuron against Cotton Leafworm Spodoptera littoralis (Boisd.). AmericanEurasian J. Agric. \& Environ. Sci., 4 (1): 62-67.

Sherief, E.A.H.; Saadia A. Abd- El-Basier and S.H.A. Hussein (2009). The Economic Injury Level of Spodoptera Littoralis (Bosid.) And Toxic Effect of Certain Compounds on Sugarbeet at Fayoum Governorate. Egypt. J. of Appl. Sci., 24(8B): 683-693. 
Said, A. A. A. et al.

تقدير كفـاءة بعض المبيدات الحشـرية ضـد دودة ورق القطن على نباتـات بنجـر

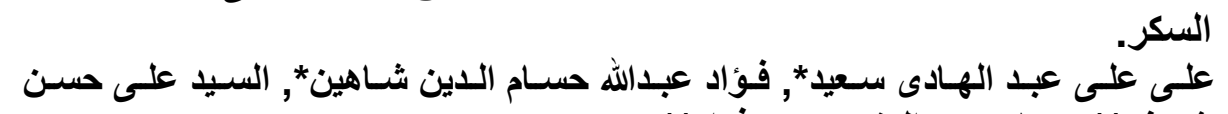

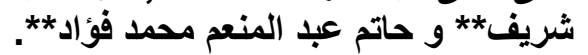

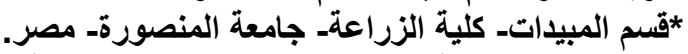

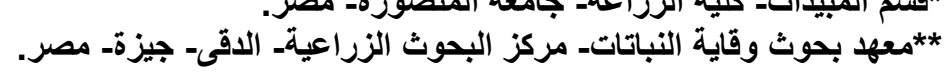

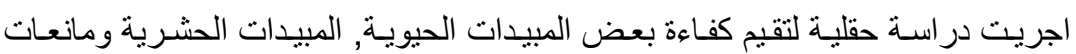

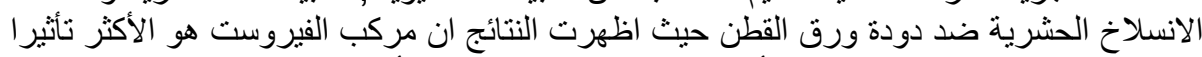

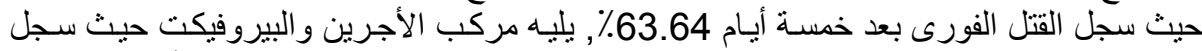
كلا منهما 51.61\% و 46.38\% خفض في التعداد. بينما كان مركب البيروتكتو هو الأخير من حيث سجل نسبة خفض 27.14\% فقط.

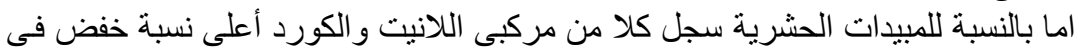

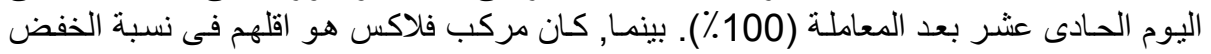
\%83.08

فى حين اخر اظهرت مانعات الانسلاخ الحشرية النتائج التالية حيث كان مركبى التوبرون والرنـر

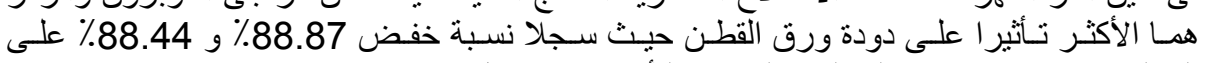

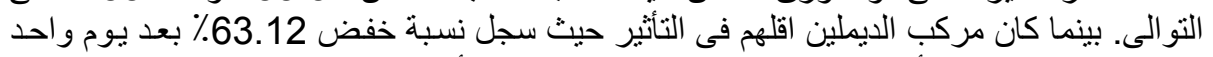

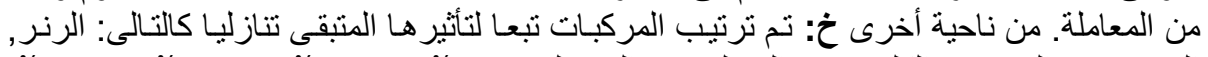

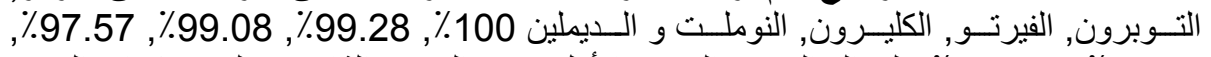

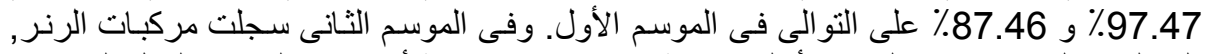

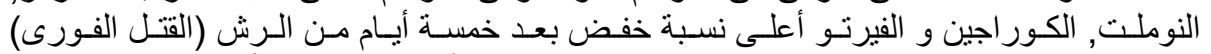

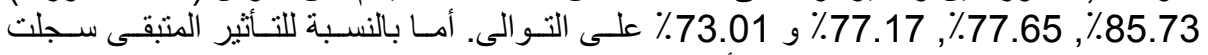

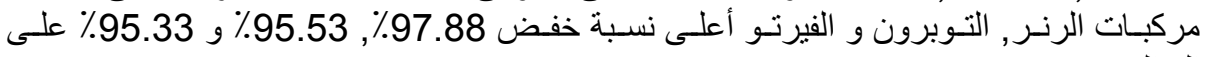

قام بتحكيم البحث

كلية الزراعة - جامعة المنصورة مركز البحوث الزراعية
أ.د / أدادل عبد المنعم صالح أ.د / وحيد محمود حسين دسوقى عيد 
J. Plant Prot. and Path., Mansoura Univ., Vol. 3 (12), December, 2012 
Table (1): Efficiency of tested biocides against larvae of Spodoptera littoralis on sugar beet leaves during 2009/2010 at Sharkia Governorate.

\begin{tabular}{|c|c|c|c|c|c|c|c|c|c|c|c|c|}
\hline \multirow{4}{*}{ Treatments } & \multicolumn{9}{|c|}{ \%Reduction after spraying } & \multirow{4}{*}{$\begin{array}{l}\text { Mean of } \\
\text { residual } \\
\text { effect }\end{array}$} & \multirow{4}{*}{$\begin{array}{l}\text { General } \\
\text { mean of \% } \\
\text { reduction }\end{array}$} & \multirow{4}{*}{$\begin{array}{l}\text { L.S.D } \\
0.05\end{array}$} \\
\hline & \multirow{3}{*}{$\begin{array}{c}\begin{array}{c}\text { before } \\
\text { application }\end{array} \\
\text { Mean }\end{array}$} & \multirow{2}{*}{\multicolumn{2}{|c|}{$\begin{array}{l}\text { Initial effect after } \\
\text { five day }\end{array}$}} & \multicolumn{6}{|c|}{ Residual effect } & & & \\
\hline & & & & ( & & $\mathrm{s}$ & & \multicolumn{2}{|c|}{11} & & & \\
\hline & & Mean & $\% R$ & Mean & $\% \mathbf{R}$ & Mean & \%R & Mean & $\% \mathbf{R}$ & & & \\
\hline Agerin & $\begin{array}{c}15.50 \mathrm{~A}^{\mathrm{a}} \\
\pm 3.10 \\
\end{array}$ & $\begin{array}{c}7.50 \mathrm{BC}^{6} \\
\pm 2.51 \\
\end{array}$ & 51.61 & $\begin{array}{c}5.25 \mathrm{~B}^{\mathrm{bc}} \\
\pm 2.75\end{array}$ & 66.13 & $\begin{array}{c}4.25 \mathrm{~B}^{\mathrm{bc}} \\
\pm 2.36\end{array}$ & 72.58 & $\begin{array}{l}3.25 \mathrm{~B}^{\mathrm{c}} \\
\pm 1.70\end{array}$ & 75.6 & 74.09 & 53.18 & $3.92^{* *}$ \\
\hline Protecto & $\begin{array}{c}17.50 \mathrm{~A}^{\mathrm{a}} \\
\pm 6.55\end{array}$ & $\begin{array}{c}12.75 \mathrm{~A}^{\mathrm{ab}} \\
\pm 6.39\end{array}$ & 27.14 & $\begin{array}{c}6.50 \mathrm{~B}^{\mathrm{bc}} \\
\pm 3.69\end{array}$ & 62.86 & $\begin{array}{l}4.25 \mathrm{~B}^{\mathrm{c}} \\
\pm 0.95\end{array}$ & 75.71 & $\begin{array}{l}3.25 \mathrm{~B}^{\mathrm{c}} \\
\pm 0.95\end{array}$ & 78.39 & 77.05 & 48.82 & $7.24^{\star *}$ \\
\hline Virust & $\begin{array}{l}14.25 \mathrm{~A}^{\mathrm{a}} \\
\pm 2.50 \\
\end{array}$ & $\begin{array}{l}5.00 \mathrm{C}^{\mathrm{b}} \\
\pm 2.16 \\
\end{array}$ & 63.64 & $\begin{array}{l}3.50 \mathrm{~B}^{\mathrm{b}} \\
\pm 2.51 \\
\end{array}$ & 74.55 & $\begin{array}{l}3.25 \mathrm{~B}^{\mathrm{b}} \\
\pm 2.62 \\
\end{array}$ & 76.36 & $\begin{array}{l}2.75 \mathrm{~B}^{\mathrm{b}} \\
\pm 2.98 \\
\end{array}$ & 76.72 & 76.54 & 58.98 & $3.77^{\star \star}$ \\
\hline Brofect & $\begin{array}{c}17.25 \mathrm{~A}^{\mathrm{a}} \\
\pm 3.40\end{array}$ & $\begin{array}{c}9.25 \mathrm{BC}^{\mathrm{b}} \\
\pm 2.75\end{array}$ & 46.38 & $\begin{array}{c}6.50 \mathrm{~B}^{\mathrm{bc}} \\
\pm 3.10\end{array}$ & 62.32 & $\begin{array}{c}5.00 \mathrm{~B}^{\mathrm{bc}} \\
\pm 3.46\end{array}$ & 71.01 & $\begin{array}{l}3.75 \mathrm{~B}^{\mathrm{C}} \\
\pm 4.19\end{array}$ & 74.7 & 72.85 & 52.04 & $4.75^{\star \star}$ \\
\hline Control & $\begin{array}{l}16.00 \mathrm{~A}^{\mathrm{a}} \\
\pm 2.16\end{array}$ & $\begin{array}{c}16.00 \mathrm{~A}^{\mathrm{a}} \\
\pm 2.16\end{array}$ & - & $\begin{array}{c}16.00 \mathrm{~A}^{\mathrm{a}} \\
\pm 2.16\end{array}$ & - & $\begin{array}{c}16.00 \mathrm{~A}^{\mathrm{a}} \\
\pm 2.16\end{array}$ & - & $\begin{array}{c}13.75 \mathrm{~A}^{\mathrm{a}} \\
\pm 2.98\end{array}$ & - & - & - & 3.44 N.S \\
\hline \begin{tabular}{|l|} 
L.S.D \\
0.05
\end{tabular} & 5.84 N.S & $5.39^{*}$ & - & $4.36^{* *}$ & - & $3.69^{\star *}$ & - & $4.22^{\star *}$ & - & - & - & - \\
\hline
\end{tabular}

${ }^{\star}$ Means followed the same capital letter in a column for different pesticides or small letter in row of each pesticides at different times are not significantly different at $5 \%$ level of Probability (Duncan's Multiple Rang Test).

Table (2): Efficiency of tested insecticides against larvae of Spodoptera littoralis on sugar beet leaves during 2009/2010 at Sharkia Governorate.

\begin{tabular}{|c|c|c|c|c|c|c|c|c|c|c|c|c|c|c|}
\hline \multirow{4}{*}{ Treatments } & \multicolumn{11}{|c|}{ \%Reduction after spraying } & \multirow{4}{*}{$\begin{array}{c}\text { Mean of } \\
\text { residual } \\
\text { effect }\end{array}$} & \multirow{4}{*}{$\begin{array}{c}\text { General } \\
\text { mean of } \\
\% \\
\text { reduction }\end{array}$} & \multirow{4}{*}{$\begin{array}{l}\text { L.S.D } \\
0.05\end{array}$} \\
\hline & \multirow{3}{*}{$\begin{array}{c}\begin{array}{c}\text { before } \\
\text { application }\end{array} \\
\text { Mean }\end{array}$} & \multirow{2}{*}{\multicolumn{2}{|c|}{\begin{tabular}{|c|} 
Initial effect \\
after two day
\end{tabular}}} & \multicolumn{8}{|c|}{ Residual effect } & & & \\
\hline & & & & $\mathbf{5}$ & & 7 & & $\mathbf{9}$ & & \multicolumn{2}{|l|}{11} & & & \\
\hline & & Mean & $\% \mathbf{R}$ & Mean & $\% \mathbf{R}$ & Mean & \%R & Mean & \%R & Mean & \%R & & & \\
\hline Lannate & $\begin{array}{l}60.5 \mathrm{~A}^{\mathrm{a}} \\
\pm 23.23 \\
\end{array}$ & $\begin{array}{c}12.75 \mathrm{~B}^{\mathrm{b}} \\
\pm 6.99 \\
\end{array}$ & 81.04 & $\begin{array}{l}3.5 \mathrm{C}^{\mathrm{b}} \\
\pm 3.31\end{array}$ & 94.73 & $\begin{array}{l}0.75 \mathrm{C}^{\mathrm{b}} \\
\pm 1.50\end{array}$ & 98.87 & $\begin{array}{l}0.00 \mathrm{C}^{b} \\
\pm 0.00\end{array}$ & 100 & $\begin{array}{l}0.00 \mathrm{C}^{\mathrm{b}} \\
\pm 0.00\end{array}$ & 100 & 94.93 & 85.86 & $14.87^{\star *}$ \\
\hline Cord & $\begin{array}{l}50.5 \mathrm{~A}^{\mathrm{a}} \\
\pm 8.22\end{array}$ & $\begin{array}{l}14.5 \mathrm{~B}^{\mathrm{b}} \\
\pm 1.29\end{array}$ & 74.12 & $\begin{array}{l}5.25 \mathrm{C}^{\mathrm{C}} \\
\pm 2.06\end{array}$ & 90.54 & $\begin{array}{l}-1.5 \mathrm{C}^{\mathrm{c}} \\
\pm 1.29\end{array}$ & 97.29 & $\begin{array}{l}0.00 \mathrm{C}^{\mathrm{c}} \\
\pm 0.00\end{array}$ & 100 & $\begin{array}{l}0.00 \mathrm{C}^{\mathrm{C}} \\
\pm 0.00\end{array}$ & 100 & 92.39 & 83.18 & $5.26^{* *}$ \\
\hline Radical & $\begin{array}{c}58.00 \mathrm{~A}^{\mathrm{a}} \\
\pm 16.26\end{array}$ & $\begin{array}{l}27.5 \mathrm{~B}^{\mathrm{b}} \\
\pm 12.92\end{array}$ & 57.26 & $\begin{array}{c}18.75 C^{b c} \\
\pm 10.01\end{array}$ & 70.59 & $\begin{array}{c}14.5 \mathrm{BC} \mathrm{C}^{\mathrm{bC}} \\
\pm 8.18\end{array}$ & 77.26 & $\begin{array}{c}9.5 \mathrm{BC}^{\mathrm{C}} \\
\pm 6.02\end{array}$ & 84.68 & $\begin{array}{c}6.00 \mathrm{BC}^{\mathrm{C}} \\
\pm 4.54\end{array}$ & 90.19 & 75.99 & 67.21 & $15.53^{\text {** }}$ \\
\hline Flaxe & $\begin{array}{l}74.25 \mathrm{~A}^{\mathrm{a}} \\
\pm 35.35\end{array}$ & $\begin{array}{l}50.5 \mathrm{~A}^{\mathrm{ab}} \\
\pm 22.78\end{array}$ & 38.69 & $\begin{array}{l}38 \mathrm{~B}^{\mathrm{bC}} \\
\pm 20.11\end{array}$ & 53.45 & $\begin{array}{c}29.25 \mathrm{~B}^{\mathrm{bc}} \\
\pm 17.15\end{array}$ & 64.17 & $\begin{array}{c}19.75 \mathrm{~B}^{\mathrm{bc}} \\
\pm 13.57\end{array}$ & 75.13 & $\begin{array}{c}13.25 \mathrm{~B}^{\mathrm{c}} \\
\pm 9.46\end{array}$ & 83.08 & 62.90 & 55.34 & $31.75^{\star}$ \\
\hline Control & $\begin{array}{c}50.25 \mathrm{~A}^{\mathrm{a}} \\
\pm 4.50 \\
\end{array}$ & $\begin{array}{l}55.75 \mathrm{~A}^{\mathrm{a}} \\
\pm 10.40 \\
\end{array}$ & - & $\begin{array}{l}55.25 \mathrm{~A}^{\mathrm{a}} \\
\pm 10.40 \\
\end{array}$ & - & $\begin{array}{c}55.25 \mathrm{~A}^{\mathrm{a}} \\
\pm 10.93 \\
\end{array}$ & - & $\begin{array}{l}53.75 \mathrm{~A}^{\mathrm{a}} \\
\pm 11.02 \\
\end{array}$ & - & $\begin{array}{r}53 \mathrm{~A}^{\mathrm{a}} \\
\pm 11.80 \\
\end{array}$ & - & - & - & $15.21 \mathrm{NS}$ \\
\hline \begin{tabular}{|l|} 
L.S.D \\
0.05
\end{tabular} & $31.19 \mathrm{NS}$ & $19.59^{\star *}$ & - & $17.04^{\star \star}$ & - & $14.84^{* *}$ & - & $12.46^{\star \star}$ & - & $10.64^{\star *}$ & - & - & - & - \\
\hline
\end{tabular}

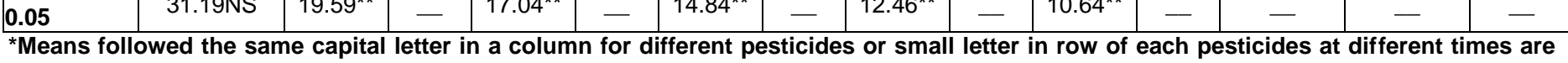
not significantly different at $5 \%$ level of Probability (Duncan's Multiple Rang Test). 
J. Plant Prot. and Path., Mansoura Univ., Vol. 3 (12), December, 2012

Table (3): Efficiency of tested pesticides against larvae of Spodoptera littoralis on sugar beet leaves during 2009/2010.

\begin{tabular}{|c|c|c|c|c|c|c|c|c|c|c|}
\hline \multirow{4}{*}{ Treatments } & \multicolumn{7}{|c|}{ \%Reduction after spraying } & \multirow{4}{*}{$\begin{array}{l}\text { Mean of } \\
\text { residual } \\
\text { effect }\end{array}$} & \multirow{4}{*}{$\begin{array}{l}\text { General } \\
\text { mean of } \% \\
\text { reduction }\end{array}$} & \multirow{4}{*}{$\begin{array}{l}\text { L.S.D } \\
0.05\end{array}$} \\
\hline & \multirow{3}{*}{$\begin{array}{c}\begin{array}{c}\text { before } \\
\text { application }\end{array} \\
\text { Mean }\end{array}$} & \multirow{2}{*}{\multicolumn{2}{|c|}{ Initial effect after five day }} & \multicolumn{4}{|c|}{ Residual effect } & & & \\
\hline & & & & & 10 & \multicolumn{2}{|c|}{15} & & & \\
\hline & & Mean & Reduct-ion & Mean & Reduct-ion & Mean & Reduct-ion & & & \\
\hline Runner & $\begin{array}{c}17.75 \mathrm{AB}^{\mathrm{a}} \\
\pm 2.06\end{array}$ & $\begin{array}{l}2.00 \mathrm{D}^{\mathrm{b}} \\
\pm 0.00\end{array}$ & 88.44 & $\begin{array}{l}0.00 \mathrm{C}^{\mathrm{C}} \\
\pm 0.00\end{array}$ & 100 & $\begin{array}{l}0.50 \mathrm{C}^{\mathrm{C}} \\
\pm 0.00\end{array}$ & 100 & 100 & 96.15 & $1.58^{* *}$ \\
\hline Nomolt & $\begin{array}{c}18.00 \mathrm{AB}^{\mathrm{a}} \\
\pm 1.70\end{array}$ & $\begin{array}{c}5.25 \mathrm{BC}^{b} \\
\pm 1.50\end{array}$ & 69.66 & $\begin{array}{l}0.75 \mathrm{C}^{\mathrm{C}} \\
\pm 0.95\end{array}$ & 94.95 & $\begin{array}{l}0.00 \mathrm{C}^{\mathrm{C}} \\
\pm 0.00\end{array}$ & 100 & 97.47 & 88.20 & $2.15^{\star *}$ \\
\hline Coragen & $\begin{array}{c}19.75 \mathrm{AB}^{\mathrm{a}} \\
\pm 1.50\end{array}$ & $\begin{array}{c}6.00 \mathrm{BC}^{\mathrm{b}} \\
\pm 2.44\end{array}$ & 68.84 & $\begin{array}{l}1.00 C^{C} \\
\pm 0.81\end{array}$ & 93.95 & $\begin{array}{c}0.75 \mathrm{BC}^{\mathrm{C}} \\
\pm 0.50\end{array}$ & 95.01 & 94.48 & 85.93 & $1.37^{\star \star}$ \\
\hline Demelin & $\begin{array}{c}22.25 \mathrm{~A}^{\mathrm{a}} \\
\pm 3.59\end{array}$ & $\begin{array}{l}8.00 \mathrm{~B}^{\mathrm{b}} \\
\pm 2.44\end{array}$ & 63.12 & $\begin{array}{l}3.25 \mathrm{~B}^{\mathrm{C}} \\
\pm 0.95\end{array}$ & 85.24 & $\begin{array}{l}1.75 \mathrm{~B}^{\mathrm{c}} \\
\pm 0.50\end{array}$ & 89.68 & 87.46 & 79.35 & $3.45^{\star *}$ \\
\hline Toporon & $\begin{array}{c}20.75 \mathrm{AB}^{\mathrm{a}} \\
\pm 4.85\end{array}$ & $\begin{array}{c}2.25 \mathrm{D}^{\mathrm{b}} \\
\pm 1.50\end{array}$ & 88.87 & $\begin{array}{l}0.25 \mathrm{C}^{\mathrm{b}} \\
\pm 0.50\end{array}$ & 98.56 & $\begin{array}{l}0.00 \mathrm{C}^{\mathrm{b}} \\
\pm 0.00\end{array}$ & 100 & 99.28 & 95.81 & $3.94^{\star *}$ \\
\hline keleron & $\begin{array}{c}18.50 \mathrm{AB}^{\mathrm{a}} \\
\pm 1.91\end{array}$ & $\begin{array}{c}5.75 \mathrm{BC} \mathrm{C}^{b} \\
\pm 0.50\end{array}$ & 68.12 & $\begin{array}{l}0.75 C^{c} \\
\pm 0.50\end{array}$ & 95.15 & $\begin{array}{l}0.00 C^{C} \\
\pm 0.00\end{array}$ & 100 & 97.57 & 87.75 & $1.57^{\star \star}$ \\
\hline Diberone & $\begin{array}{c}18.00 \mathrm{AB}^{\mathrm{a}} \\
\pm 2.82\end{array}$ & $\begin{array}{c}5.25 \mathrm{BC} \\
\pm 2.21\end{array}$ & 70.08 & $\begin{array}{c}1.50 \mathrm{BC}^{\mathrm{C}} \\
\pm 1.00\end{array}$ & 90.05 & $\begin{array}{l}1.00 \mathrm{C}^{\mathrm{C}} \\
\pm 0.95\end{array}$ & 90.99 & 90.52 & 83.70 & $2.96^{* *}$ \\
\hline Virtue & $\begin{array}{c}16.25 \mathrm{~B}^{\mathrm{a}} \\
\pm 2.21\end{array}$ & $\begin{array}{c}4.25 \mathrm{CD}^{\mathrm{b}} \\
\pm 0.95\end{array}$ & 73.17 & $\begin{array}{l}0.25 \mathrm{C}^{\mathrm{C}} \\
\pm 0.50\end{array}$ & 98.16 & $\begin{array}{l}0.00 \mathrm{C}^{\mathrm{c}} \\
\pm 0.00\end{array}$ & 100 & 99.08 & 90.44 & $1.89^{\star \star}$ \\
\hline Control & $\begin{array}{c}20.00 \mathrm{AB}^{\mathrm{a}} \\
\pm 3.36\end{array}$ & $\begin{array}{c}20.75 \mathrm{~A}^{\mathrm{a}} \\
\pm 3.30\end{array}$ & - & $\begin{array}{c}17.75 \mathrm{~A}^{\mathrm{a}} \\
\pm 3.40\end{array}$ & - & $\begin{array}{c}16.25 \mathrm{~A}^{\mathrm{a}} \\
\pm 2.36\end{array}$ & - & - & - & $4.84 \mathrm{~N} . \mathrm{S}$ \\
\hline \begin{tabular}{|l|} 
L.S.D \\
0.05
\end{tabular} & 4.20 N.S & $2.54^{\star \star}$ & - & $1.92^{\star *}$ & - & $1.25^{\star \star}$ & - & - & - & - \\
\hline
\end{tabular}


Said, A. A. A. et al.

Table (4): Efficiency of tested pesticides against larvae of Spodoptera littoralis on sugar beet leaves during 2010/2011.

\begin{tabular}{|c|c|c|c|c|c|c|c|c|c|c|}
\hline \multirow{4}{*}{ Treatments } & \multicolumn{7}{|c|}{ \%Reduction after spraying } & \multirow{4}{*}{$\begin{array}{l}\text { Mean of } \\
\text { residual } \\
\text { effect }\end{array}$} & \multirow{4}{*}{$\begin{array}{l}\text { General } \\
\text { mean of \% } \\
\text { reduction }\end{array}$} & \multirow{4}{*}{$\begin{array}{l}\text { L.S.D } \\
0.05\end{array}$} \\
\hline & \multirow{3}{*}{$\begin{array}{c}\begin{array}{c}\text { before } \\
\text { application }\end{array} \\
\text { Mean }\end{array}$} & \multirow{2}{*}{\multicolumn{2}{|c|}{$\begin{array}{c}\text { Initial effect after five } \\
\text { day }\end{array}$}} & \multicolumn{4}{|c|}{ Residual effect } & & & \\
\hline & & & & \multicolumn{2}{|r|}{ mosing } & \multicolumn{2}{|c|}{15} & & & \\
\hline & & Mean & Reduct-ion & Mean & Reduct-ion & Mean & Reduct-ion & & & \\
\hline Runner & $\begin{array}{c}36.25 \mathrm{~A}^{\mathrm{a}} \\
\pm 2.75\end{array}$ & $\begin{array}{l}5.00 \mathrm{~B}^{\mathrm{b}} \\
\pm 1.15\end{array}$ & 85.73 & $\begin{array}{l}1.25 \mathrm{C}^{\mathrm{C}} \\
\pm 0.95\end{array}$ & 95.77 & $\begin{array}{l}0.00 \mathrm{C}^{\mathrm{C}} \\
\pm 0.00\end{array}$ & 100 & 97.88 & 93.83 & $2.41^{\star *}$ \\
\hline Nomolt & $\begin{array}{c}31.25 \mathrm{ABC}^{\mathrm{a}} \\
\pm 5.96\end{array}$ & $\begin{array}{l}6.75 \mathrm{~B}^{\mathrm{b}} \\
\pm 3.50\end{array}$ & 77.65 & $\begin{array}{l}2.75 \mathrm{BC} \mathrm{C}^{\mathrm{bc}} \\
\pm 1.71\end{array}$ & 89.20 & $\begin{array}{l}0.25 \mathrm{C}^{\mathrm{c}} \\
\pm 0.50\end{array}$ & 98.79 & 93.99 & 88.55 & $5.50^{* \star}$ \\
\hline Coragen & $\begin{array}{c}34.00 \mathrm{~A}^{\mathrm{a}} \\
\pm 8.86\end{array}$ & $\begin{array}{l}7.50 \mathrm{~B}^{\mathrm{b}} \\
\pm 3.78\end{array}$ & 77.17 & $\begin{array}{c}2.75 \mathrm{BC}^{\mathrm{b}} \\
\pm 1.25\end{array}$ & 90.07 & $\begin{array}{c}1.25 \mathrm{BC}^{\mathrm{b}} \\
\pm 0.50\end{array}$ & 94.46 & 92.26 & 87.23 & $7.50^{* \star}$ \\
\hline Demelin & $\begin{array}{c}32.25 \mathrm{AB}^{\mathrm{a}} \\
\pm 8.09\end{array}$ & $\begin{array}{l}9.50 \mathrm{~B}^{\mathrm{b}} \\
\pm 3.69\end{array}$ & 69.51 & $\begin{array}{c}4.75 \mathrm{~B}^{\mathrm{b}} \\
\pm 2.21\end{array}$ & 81.93 & $\begin{array}{c}2.25 \mathrm{~B}^{\mathrm{b}} \\
\pm 1.50\end{array}$ & 89.49 & 85.71 & 80.31 & $7.16^{* \star}$ \\
\hline Toporon & $\begin{array}{c}28.25 \mathrm{ABCD}^{\mathrm{a}} \\
\pm 6.70\end{array}$ & $\begin{array}{l}9.75 \mathrm{~B}^{\mathrm{b}} \\
\pm 6.29\end{array}$ & 64.28 & $\begin{array}{l}1.75 \mathrm{BC}^{\mathrm{C}} \\
\pm 2.87\end{array}$ & 92.40 & $\begin{array}{l}0.25 \mathrm{C}^{\mathrm{C}} \\
\pm 0.50\end{array}$ & 98.66 & 95.53 & 85.11 & $7.42^{* \star}$ \\
\hline keleron & $\begin{array}{c}22.25 \mathrm{CD}^{\mathrm{a}} \\
\pm 3.77\end{array}$ & $\begin{array}{l}6.75 \mathrm{~B}^{\mathrm{b}} \\
\pm 1.50\end{array}$ & 67.13 & $\begin{array}{c}2.25 \mathrm{BC} \mathrm{bc}^{\mathrm{bc}} \\
\pm 2.21\end{array}$ & 87.01 & $\begin{array}{l}0.00 \mathrm{C}^{\mathrm{C}} \\
\pm 0.00\end{array}$ & 100 & 93.51 & 84.17 & $4.75^{\star \star}$ \\
\hline Diberone & $\begin{array}{c}19.75 \mathrm{D}^{\mathrm{a}} \\
\pm 6.39\end{array}$ & $\begin{array}{l}6.25 \mathrm{~B}^{\mathrm{b}} \\
\pm 4.03\end{array}$ & 67.25 & $\begin{array}{c}2.50 \mathrm{BC}^{\mathrm{b}} \\
\pm 1.73\end{array}$ & 84.47 & $\begin{array}{l}1.75 \mathrm{~B}^{\mathrm{b}} \\
\pm 1.25\end{array}$ & 86.65 & 85.56 & 79.45 & $6.05^{\star \star}$ \\
\hline Virtue & $\begin{array}{c}23.00 \mathrm{BCD}^{\mathrm{a}} \\
\pm 2.82\end{array}$ & $\begin{array}{l}6.00 \mathrm{~B}^{\mathrm{b}} \\
\pm 2.71\end{array}$ & 73.01 & $\begin{array}{c}1.75 \mathrm{BC}^{\mathrm{c}} \\
\pm 0.95\end{array}$ & 90.66 & $\begin{array}{l}0.00 \mathrm{C}^{\mathrm{C}} \\
\pm 0.00\end{array}$ & 100 & 95.33 & 87.88 & $3.10^{* \star}$ \\
\hline Control & $\begin{array}{l}29.75 \mathrm{ABC}^{\mathrm{a}} \\
\quad \pm 1.71\end{array}$ & $\begin{array}{c}28.75 \mathrm{~A}^{\mathrm{a}} \\
\pm 1.71\end{array}$ & - & $\begin{array}{c}24.25 \mathrm{~A}^{\mathrm{b}} \\
\pm 1.71\end{array}$ & - & $\begin{array}{c}19.75 \mathrm{~A}^{\mathrm{C}} \\
\pm 1.25\end{array}$ & - & - & - & $2.47^{* \star}$ \\
\hline $\begin{array}{l}\text { L.S.D } \\
0.05\end{array}$ & $8.58^{*}$ & $5.07^{* *}$ & - & $2.66^{* *}$ & - & $1.20^{\star *}$ & - & - & - & - \\
\hline
\end{tabular}

"Means followed the same capital letter in a column for different pesticides or small letter in row of each pesticides at different times are not significantly different at $5 \%$ level of Probability (Duncan's Multiple Rang Test). 